\title{
On Weak-Field (One-Photon) Coherent Control of Photoisomerization
}

\section{Dey, Diptesh; Henriksen, Niels Engholm}

Published in:

Journal of Physical Chemistry Letters

Link to article, DOI:

10.1021/acs.jpclett.0c02273

Publication date:

2020

Document Version

Peer reviewed version

Link back to DTU Orbit

Citation (APA):

Dey, D., \& Henriksen, N. E. (2020). On Weak-Field (One-Photon) Coherent Control of Photoisomerization. Journal of Physical Chemistry Letters, 11, 8470-8476. https://doi.org/10.1021/acs.jpclett.0c02273

\section{General rights}

Copyright and moral rights for the publications made accessible in the public portal are retained by the authors and/or other copyright owners and it is a condition of accessing publications that users recognise and abide by the legal requirements associated with these rights.

- Users may download and print one copy of any publication from the public portal for the purpose of private study or research.

- You may not further distribute the material or use it for any profit-making activity or commercial gain

- You may freely distribute the URL identifying the publication in the public portal

If you believe that this document breaches copyright please contact us providing details, and we will remove access to the work immediately and investigate your claim 


\title{
On Weak-Field (One-Photon) Coherent Control of Photoisomerization
}

\author{
Diptesh Dey and Niels E. Henriksen* \\ Department of Chemistry, Technical University of Denmark, Building 207, DK-2800 \\ Lyngby, Denmark \\ E-mail: neh@kemi.dtu.dk
}

*To whom correspondence should be addressed 


\begin{abstract}
Photochemistry induced by phase-coherent laser light is an intriguing topic. The possibility of weak-field (one-photon) phase-only control of photoisomerization is controversial. Experimental studies on the weak-field coherent control of cis-trans isomerization have lead to conflicting results. Here we address this issue by quantum dynamical calculations, focusing on a mechanism where different "phase-shaped" wave packets are quickly stabilized ("dumped") in the trans configuration due to prompt energy dissipation. We systematically investigate different relaxation rates with the system-bath dynamics described within the time-dependent-Hartree approximation leading to a friction-type force. We find evidence for phase control of trans-isomer yields (about $5 \%$ ) in this model with pure energy dissipation given sufficiently strong dampening.
\end{abstract}

\title{
TOC Graphic
}

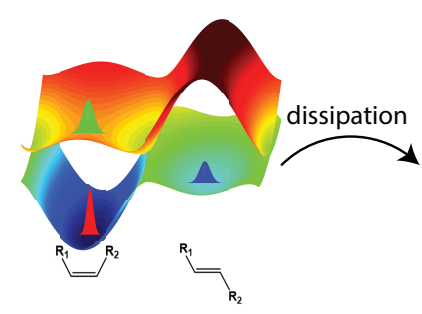

Introduction. The phase coherence of laser light can lead to coherent excitation of matter and hence control over the quantum mechanical interference effects in molecules. ${ }^{1-8}$ This is an extension of traditional photochemistry with incoherent light. Control is exerted by laser pulse shaping, e.g., by modulating the phases of each spectral component of an ultrashort laser pulse.

Early theoretical considerations ruled out one-photon coherent control of photofragmentation. ${ }^{9}$ To be more specific, in the weak-field (one-photon) limit for gas-phase photofragmentation of isolated molecules with the molecule initially in an eigenstate of the ground electronic state, it has been shown that in the long-time limit all observables associated with 
the fragments are independent of the spectral phases of the laser light. ${ }^{9-12}$ That is, for this particular scenario the phase coherence of laser light plays no role.

Although coherent control is not possible in the long-time limit where dissociation is completed, we have demonstrated that phase-modulation of the outcome of "early" dynamics is possible. Thus, transient post-pulse phase effects on the total dissociation probability, the final vibrational states of fragments, and electronic branching ratios have been predicted. ${ }^{12-14}$ The transient effects are particularly "long-lived" for indirect dissociation where an intermediate energized complex is formed, i.e., products may then continuously "leak out" of the complex over an extended period of time and a phase dependence on the "early" products can be observed. Furthermore, it should be noted that coherent weak-field control of photofragmentation out of nonstationary coherent superpositions of eigenstates is possible, also in the long-time limit. ${ }^{15,16}$

Besides photofragmentation, weak-field phase control has been predicted to be possible in the long-time limit, e.g., for photoisomerization. ${ }^{11}$ To that end, experimental studies on weakfield (one-photon) coherent control of the cis-trans isomerization of the retinal chromophore embedded in a protein environment (e.g. rhodopsin) have lead to conflicting results. ${ }^{17,18}$ Thus, based on these experimental works the question as to whether the weak-field coherent control of photoisomerization is possible, is still unclear. However, it should be added that the observed effect was quite modest, about $4 \%$ yield difference upon changing laser phase. ${ }^{17}$

Seminal experimental and theoretical studies on the femtosecond dynamics of cis-trans photoisomerization includes Refs. ${ }^{19-28}$ We note in particular that an appropriate theoretical description of the photo-induced isomerization must account for (intramolecular) vibrational cooling. ${ }^{20,24}$ In the absence of a cooling mechanism for the reaction coordinate, the molecule will continuously oscillate back and forth between the cis and trans configurations.

On the theoretical side, substantial weak-field coherent control has been predicted for a simple one-dimensional model of isomerization where the two isomers are represented by two non-adiabatically coupled Morse potentials. ${ }^{29}$ Thus, it was suggested that if the timescale of 
non-adiabatic population transfer between the isomers becomes comparable to the timescale of environmentally assisted energy relaxation, an opportunity for coherent control emerges. A quantum mechanical study based on a more realistic model Hamiltonian representing 26degrees of freedom in the retinal molecule ${ }^{30}$ showed a small effect (less than 10\%) of laser chirp in a time window which was limited to 2 ps. To that end, it should be noted that this time window did not allow for a complete stabilization of the cis/trans populations.

In the present work, it is not our intention to attempt exact numerical modelling of a specific system (like retinal) but rather to study more generic dynamics using a model with some physically reasonable parameters. We have used a generic two-degrees of freedom model for the cis-trans isomerization. ${ }^{22}$ This model is extended by a friction-type interaction with a heat bath. We present a systematic investigation of how the quantum yield of the trans product depends on laser chirp (phase-only control) and on the rate of energy relaxation in the reaction coordinate.

Theory. We model the cis-trans photoisomerization with a two-dimensional Hamiltonian as originally suggested by Stock and Hahn. ${ }^{22}$ This semi-empirical model consists of two nonadiabatically coupled electronic states $S_{0}$ and $S_{1}$ with a reaction coordinate $\varphi$, describing the torsional motion of isomerization and a vibronically active vibrational mode $q .{ }^{21}$ This model reproduces many salient features observed in the time-resolved experiments of the lightinduced photoisomerization of the retinal chromophore embedded in the protein environment of rhodopsin. ${ }^{19}$

In the diabatic electronic-state representation, the system Hamiltonian in mutually orthogonal coordinates, $\varphi$ and $q$ reads (atomic units are used unless otherwise stated):

$$
\hat{H}(s)=\hat{T} \mathbf{I}+\left(\begin{array}{ll}
V_{00} & V_{01} \\
V_{10} & V_{11}
\end{array}\right),
$$


where $s=\{\varphi, q\}$ and the kinetic energy operator

$$
\hat{T}=-\frac{1}{2 \mathrm{I}} \frac{\partial^{2}}{\partial \varphi^{2}}-\frac{\omega_{q}}{2} \frac{\partial^{2}}{\partial q^{2}}
$$

with the reduced moment of inertia I. The elements of the diabatic potential matrix represented in a basis spanned by the diabatic electronic wave functions are

$$
\begin{gathered}
V_{00}=\frac{1}{2} W_{0}(1-\cos \varphi)+\frac{1}{2} \omega_{q} q^{2}, \\
V_{11}=E_{1}-\frac{1}{2} W_{1}(1-\cos \varphi)+\kappa q+\frac{1}{2} \omega_{q} q^{2},
\end{gathered}
$$

and

$$
V_{01}=V_{10}=\lambda[\sin \varphi]^{2} q
$$

where $\omega_{q}$ is the frequency of the vibrational mode, $\kappa$ introduces a shift in the excited-state potential energy surface with respect to the ground-state geometry at $q=0$, and $\lambda$ is the vibronic coupling constant of the diabatic coupling. The diabatic potentials $V_{00}$ and $V_{11}$ intersect at $\varphi=\pi / 2$. Figure 1 shows the corresponding adiabatic potentials with conical intersections at $\varphi=\pi / 2+n \pi, q=0$. In the present study, we have slightly modified the original model potential by introducing a $\varphi$-dependent coupling in $V_{01}=V_{10}$ such that now the coupling is largest at the conical intersections. The parameters of the model Hamiltonian in Eq. (1) are chosen similar to Refs. ${ }^{22,23}$

The state of the system as a function of time is expressed in terms of the diabatic nuclear wave functions $\chi_{0}(\varphi, q ; t)$ and $\chi_{1}(\varphi, q ; t)$ as,

$$
|X(\varphi, q ; t)\rangle=\left(\begin{array}{c}
\chi_{0}(\varphi, q ; t) \\
\chi_{1}(\varphi, q ; t)
\end{array}\right)
$$


which is obtained by solving the time-dependent Schrödinger equation,

$$
i \frac{\partial}{\partial t}|X(\varphi, q ; t)\rangle=\hat{H}(s)|X(\varphi, q ; t)\rangle
$$

The interaction of the molecular system with the radiation field is described within the

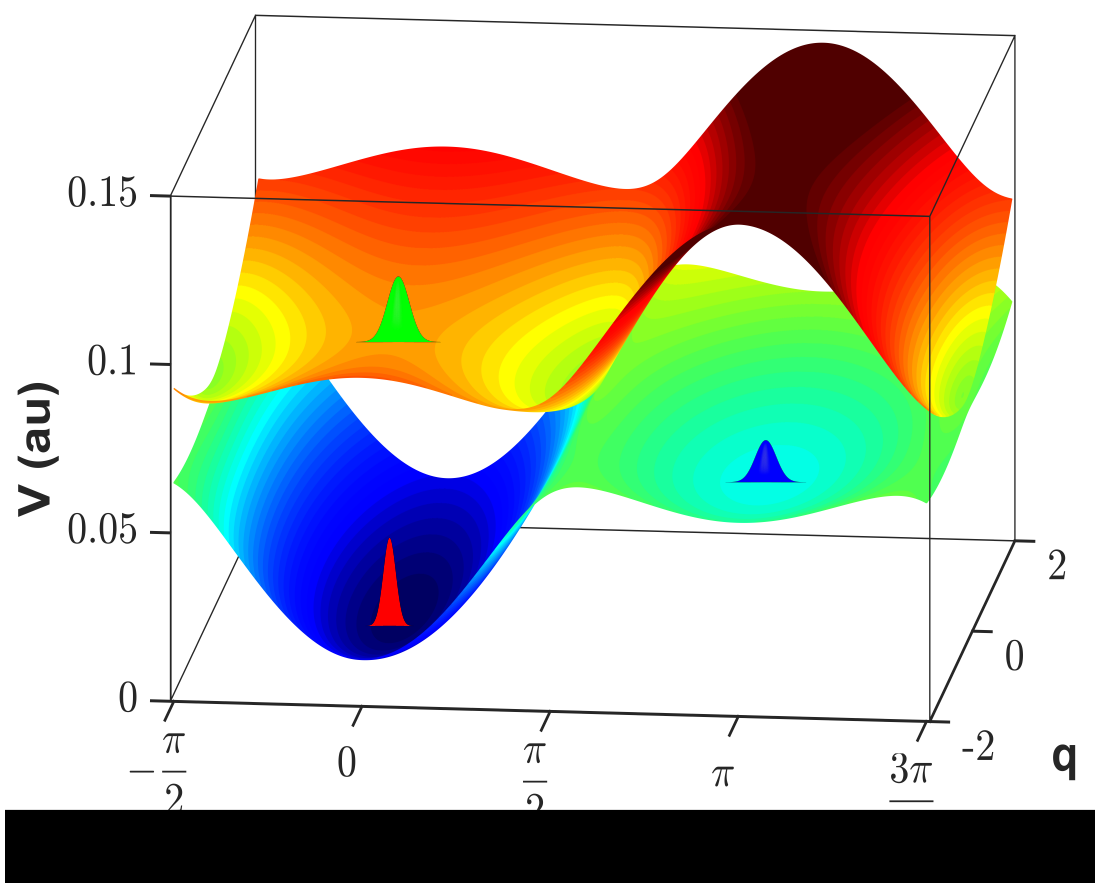

Figure 1: The adiabatic potential energy surfaces for the $\operatorname{cis}(\varphi=0)$ to trans $(\varphi=\pi)$ transformation. The nuclear wave packets are sketched for the initial state, a state shortly after vertical excitation, and a fully relaxed trans configuration.

electric-dipole approximation,

$$
\hat{H}_{I}=-E(t)\left(\begin{array}{cc}
0 & \mu_{01} \\
\mu_{10} & 0
\end{array}\right) \text {, }
$$

where the electric transition-dipole moment function is assumed to be constant $\left(\mu_{01}=\mu_{10}\right.$ $=1 \mathrm{au}$ ) as a function of the nuclear coordinates (Condon approximation). The electric field 
of the laser pulse can be represented by

$$
E(t)=E_{0} \operatorname{Re}\left\{\int_{-\infty}^{\infty} A(\omega) e^{i \phi(\omega)} e^{-i \omega t} d \omega\right\}
$$

where $A(\omega)$ is the amplitude and $\phi(\omega)$ the phase of the frequency component at frequency $\omega$. For later reference, it is instructive to consider the interaction with such a field in the weakfield limit. The nuclear state $\left|\chi_{1}\right\rangle$, created in a weak-field bound-free transition (described by first-order perturbation theory) from the initial stationary nuclear state $\left|\chi_{0}\right\rangle$ in the lower electronic state with energy $\epsilon_{0}$, is ${ }^{31}$

$$
\left|\chi_{1}(t)\right\rangle=E_{0}(i \pi / \hbar) \int A\left(\omega_{E}\right) e^{i \phi\left(\omega_{E}\right)}\langle E \mid \phi\rangle|E\rangle e^{-i E t / \hbar} d E
$$

for times $t$ where the pulse has vanished, where $|\phi\rangle=\mu_{01}\left|\chi_{0}\right\rangle$ with $\mu_{01}$ being the projection of the electronic transition dipole moment on the polarization vector of the electric field, and $\omega_{E}=\left(E-\epsilon_{0}\right) / \hbar$. Thus, the energy as well as the energy spread of the wave packet is controlled by the frequency distribution $A(\omega)$ (bandwidth) of the laser pulse whereas the phase of each spectral component of the field translates directly into the phase of each expansion coefficient of the wave packet. That is, phase-only laser pulse shaping leads to the creation of a "phase-shaped" wave packet. We note that subsequent non-adiabatic wavepacket transition probabilities are independent of the phases $\phi(\omega)$ (see, e.g. Ref. ${ }^{14}$ ).

Energy relaxation or dissipation leading to (intramolecular) vibrational cooling can be described as being due to coupling to secondary degrees of freedom, i.e., a bath. For a sufficiently small number of degrees of freedom, a numerically exact approach like MCTDH (MultiConfigurational Time Dependent Hartree) can be applied to the entire system including the bath. ${ }^{30,32}$ Typically, however, approximate approaches like the Redfield description are applied. ${ }^{24,33}$ The Redfield approach is based on several approximations (like second-order perturbation theory for the interaction between the system and the bath and the Markov approximation) and we note in passing that the validity of the Redfield approach has been 
examined in direct comparisons with MCTDH calculations. ${ }^{32}$ Note also that previous work suggests that the possibility of weak-field control is destroyed by the Markov approximation. ${ }^{30,34}$

In this exploratory study, we want to investigate whether pure energy dissipation can lead to phase control in the weak-field limit. To that end, we start from the Time-Dependent Hartree (TDH) approximation for the system-bath coupling which is also often referred to as the Time-Dependent-Self-Consistent-Field (TDSCF) approximation. ${ }^{35,36}$ This approach omits effects of quantum entanglement between the system and the bath due to the enforced product form of the total wave function. However, it leads to a qualitative correct description of pure energy dissipation allowing us to make contact with a simple physical picture of the mechanism. As detailed in the Supporting Information (SI), dissipation is described by the addition of an appropriate time-dependent potential to each of the diabatic potentials. Thus, to $V_{00}$ and $V_{11}$ in Eq. (1) we add, respectively, $V_{0}(\varphi, t)=F_{0}(t) Q(\varphi)$ and $V_{1}(\varphi, t)=F_{1}(t) Q(\varphi)$, where the time-dependent function $F_{i}(t)$ is chosen such (see Eq. (S7) of the SI) that energy dissipation is obtained in each electronic state and $Q(\varphi)$ is the $\varphi$ dependence in the systembath interaction potential.

As a simple example, consider the dynamics in a single electronic state (without nonadiabatic coupling) and a linear interaction in the system coordinate, $Q(s)=s$. Then (see Eq. (7) of the SI) $F(t)=\lambda_{d}\langle\hat{p}\rangle / m$ and the time-dependent potential becomes $V(s, t)=$ $F(t) Q(s)=\lambda_{d}(\langle\hat{p}\rangle / m) s$, where $\lambda_{d}>0$ is a free parameter which determines the strength of the system-bath coupling. This result is similar to the classical frictional force $-\lambda_{d} v$ dependent on the velocity $v$. Applied to a harmonic oscillator, it shows that efficient energy dissipation is obtained for a time-dependent force with a frequency which matches the oscillator frequency, but is out of phase with the vibrational motion. Note that the energy dissipation is determined by the expectation values of the velocity, i.e., independent of the detailed shape of the wave packet.

Computational Details. The numerically exact ground-state wave packet corresponding 
to the initial state of the system is first prepared in the cis-configuration $(\varphi=0)$ of the $S_{0}$-state by the imaginary time propagation method. ${ }^{37}$ A regular grid of $2^{10}=1024$ grid points are taken for both $\varphi$ and $q$ coordinates; where $\varphi$ varies from zero to $2 \pi$ and the range of oscillations along the $q$ coordinate was found to be accurately described within $|q|<6$ au.

We photoexcited the molecule with a linearly chirped Gaussian femtosecond pulse, $E(t)$, as described in Refs. ${ }^{16,31,38}$ The phase function in Eq. (9) is $\phi(\omega)=\beta_{0}\left(\omega-\omega_{0}\right)^{2} / 2$, i.e., quadratic around the central frequency $\omega_{0}$. The corresponding wavelength is choosen to be $500 \mathrm{~nm}$. The peak intensity of the laser pulse was varied from $1.0 \times 10^{6}$ to $1.0 \times 10^{11}$ $\mathrm{W} \mathrm{cm}^{-2}$ to ensure the linear excitation regime. With intensities within the linear (onephoton) regime, we obtain excitation probabilities around $1 \%$. We then introduce dissipation in the $\varphi$ coordinate by the addition of the $F_{i}(t) Q(\varphi)$ potential in each diabatic state. For the system-bath coupling, we choose $Q(\varphi)=2.0+\sin (\varphi)+\cos (\varphi)$ (see also Ref. ${ }^{24}$ ).

Upon photo-excitation, the $S_{0} \rightarrow S_{1}$ transition is induced, creating a non-stationary excited-state population. The time-evolution of the wave packet is calculated by the splitoperator method ${ }^{39}$ in combination with the fast Fourier transform (FFT) algorithm. ${ }^{40}$ The FFT is exploited both along the $\varphi$ and $q$ coordinates, the former satisfying the periodic boundary condition $\Phi(\varphi)=\Phi(\varphi+2 \pi)$. The relation between the associated transforms along $\varphi$ and $q$ can be obtained from Ref. ${ }^{41}$

The nonadiabatic numerical simulation is carried out by analytical diagonalization of the $2 \times 2$ potential matrix in addition to the forward and backward transformation of the wave packet from position and momentum representations for the evaluation of the kinetic energy operator, for each coordinate and at every time step. ${ }^{42}$ Convergence of the results is ensured by choosing a time step of 0.125 au.

The time-dependent photoreaction quantum yield of the trans isomer is then calculated from the fraction of the initially excited population which is accumulated in the ground 
adiabatic state in the long-time limit as,

$$
Y=\frac{P_{\text {trans }}^{(1)}(t)}{P_{\text {cis }}^{(0)}(t)+P_{\text {trans }}^{(1)}(t)} \quad(t \rightarrow \infty)
$$

where, $P_{\text {cis }}^{(0)}(t)$ and $P_{\text {trans }}^{(1)}(t)$ refer to the time-dependent populations of the cis and trans isomers associated with the diabatic electronic states $V_{00}$ and $V_{11}$ in the regions of the potential surface $\varphi \in[-\pi / 2, \pi / 2]$ and $\varphi \in[\pi / 2,3 \pi / 2]$, respectively, as indicated in Figure 1 .

Results and Discussion. The Franck-Condon wave packet is born at $(\varphi=0, q=-0.5)$ close to the saddle point of the upper adiabatic state and the initial motion along the $\varphi$ angle is due to wave packet spreading. At the same time, the wave packet oscillates in the $q$ coordinate with a period of around 20 fs. For short-pulse excitation, the intersection region is reached in less than 100 fs and the wave packet splits into two with one part showing up in the "trans-well" of the lower adiabatic state. It should be noted that due to the rotational symmetry of the potential, the wave packet enters the "trans-well" simultaneously at $\varphi=-\pi / 2$ and $\varphi=\pi / 2$. The first maximum in the trans population is reached after about 170 fs. This part can now oscillates back and forth and visit either the "cis-well" of the lower or upper adiabatic state.

Here we investigate a mechanism similar to the one proposed in Ref. ${ }^{29}$ Thus, a short pump-pulse creates either a focused or de-focused wave packet at the crossing - depending on the sign of the chirp. ${ }^{38}$ This wave packet is subsequently stabilized ("dumped") in the trans configuration due to energy relaxation. In order to demonstrate phase-only control, the energy relaxation has to act on a fast timescale (comparable to the timescale of the nonadiabatic population transfer) and discriminate between the focused and de-focused wave packets.

We consider two pulses with linear temporal chirp: (I) a pulse where the pulse duration of the chirped pulse is less than the time it takes to reach the intersection; the peak intensity is $I_{0}=4.0 \times 10^{9} \mathrm{~W} \mathrm{~cm}^{-2}$ with a full-width-half-maximum (FWHM) of the transform-limited 
pulse of $\tau_{\mathrm{FWHM}}^{0}=20 \mathrm{fs}$ and a quadratic spectral chirp of $\beta_{0}= \pm 200 \mathrm{fs}^{2}$ leading to a pulse duration of $\tau_{\mathrm{FWHM}}=59.0 \mathrm{fs}$, and (II) the pulse considered previously by Arango and Brumer $^{30}$ where the pulse duration of the chirped pulse is $\tau_{\mathrm{FWHM}}=200 \mathrm{fs}$ with a quadratic spectral chirp of $\beta_{0}= \pm 2512 \mathrm{fs}^{2}$ corresponding to a transform-limited pulse of $\tau_{\text {FWHM }}^{0}=75.2$ fs, and for the peak intensity of the transform-limited pulse, we choose $I_{0}=8.9 \times 10^{8} \mathrm{~W} \mathrm{~cm}^{-2}$. For pulse (I) compared to the unchirped pulse, the negative chirp leads to a focused wave packet at the intersection whereas the positive chirp leads to a defocused wave packet. Note that only the phases of the wave-packet expansion coefficients are affected by the chirp (see Eq. (10)) whereas the energy and energy spread remains unchanged.

The quantum yields obtained for pulse (I), do not differ appreciably between the chirped and unchirped pulses. Thus, we focus on pulse (II). Figure 2 shows the quantum yield for the undampened system (note that the reported yields are meaningful only after the laser pulse has vanished, i.e., for $t>200 \mathrm{fs}$ ). For the transform-limited pulse, we see fairly regular oscillations in the yield with a period of about 275 fs corresponding to motion along the $\varphi$ coordinate. We observe a substantial effect of the chirp. The yield for the negative chirp is relatively constant within the first ps, in contrast to the yields obtained with the unchirped or positively chirped pulses. Note also that the initial rise in the quantum yield is delayed for the negatively chirped pulse in agreement with the focused nature of this wave packet. Figure 3 shows a contour plot of the wave packet at $t=250 \mathrm{fs}$ right after the (chirped) pulse has vanished. The (instantaneous) yields $t=250$ fs are 0.25 and 0.41 for the positively and negatively chirped pulses, respectively. It is clear that the structure of the wave packet is complicated. To that end, it must be recalled that the non-adiabatic coupling depends on the fast motion in the vibrational coordinate $q$ and that wave packet interference is created due to the rotational symmetry. Furthermore, the wave packet motion is unbound in the rotational coordinate.

Next we consider the curve crossing coupled to a heat bath, i.e., including energy dissipation. It is clear that if we could "freeze" the instantaneous populations at $t=250$ fs by a 


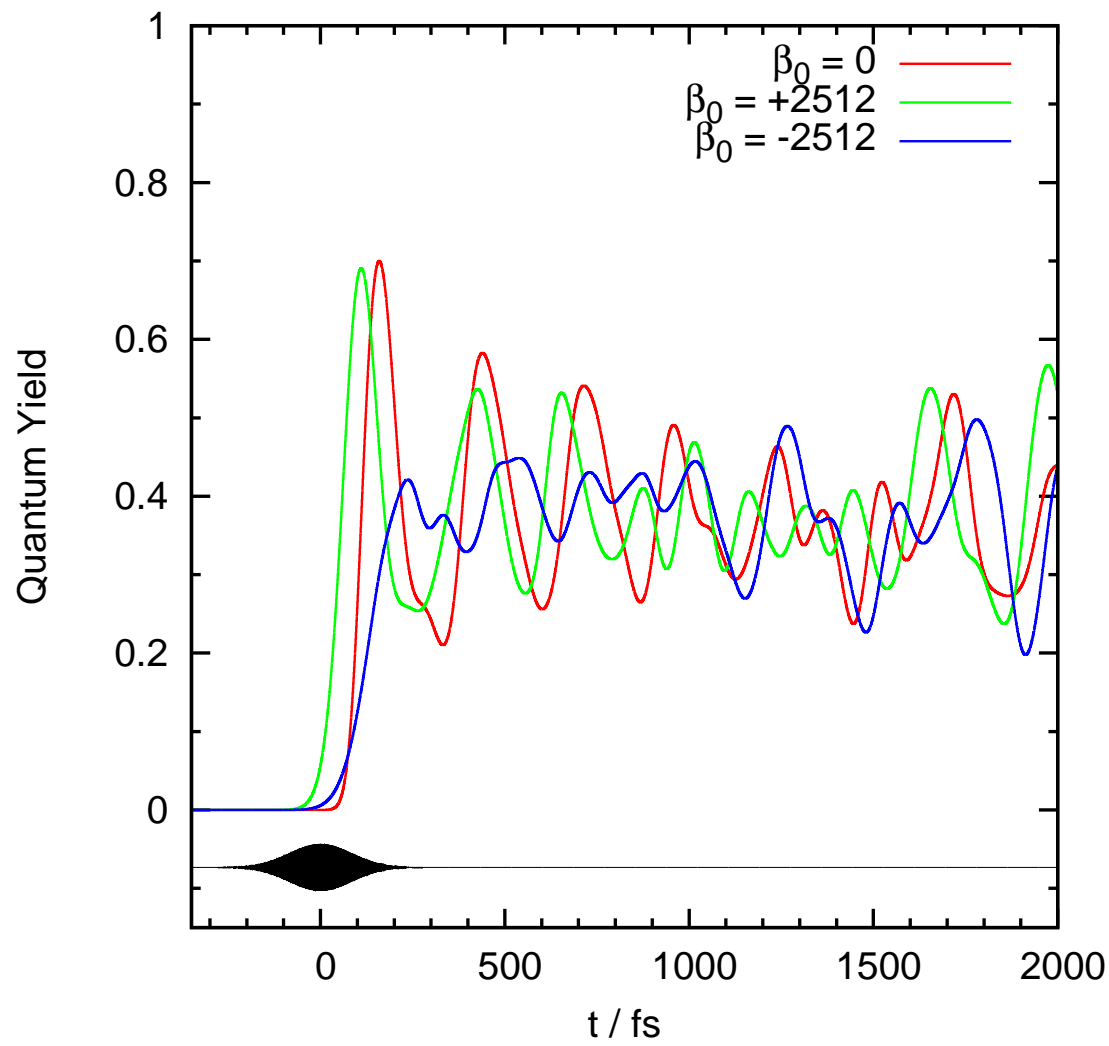

Figure 2: The quantum yield of the trans isomer calculated with the two-dimensional Hamiltonian in the absence of the heat bath. The yield is shown for the transform-limited pulse $\left(\beta_{0}=0\right)$ as well as for two chirped pulses with the electric field peaked at $t=0$ (shown for the chirped pulses).

prompt energy dissipation such that the wave packet gets trapped below the barrier for the cis-trans isomerization of the $S_{0}$ state, then weak-field (one-photon) coherent control would be demonstrated. We consider the chirped pulses described above for different strengths of the energy dissipation. Figure 4 shows the quantum yield when $\lambda_{d}=0.05$ (in Eq.(7) of the SI). Compared to Figure 2 for the undampened system, we observe a clear stabilization of the yields, especially after around 1 ps. The fast small-amplitude oscillations are related to the dampening mechanism, similar oscillations have been observed previously. ${ }^{29}$ Clearly, the quantum yield of the trans isomer is not fully converged on the timescale of 2 ps and the dependence on the chirp is small and without a systematic trend. In practice, the yield will be detected with a finite time resolution. To that end, we show in Figure 4 also the yields convoluted with a 100 fs (FWHM) Gaussian probe pulse. Again, a systematic dependence 


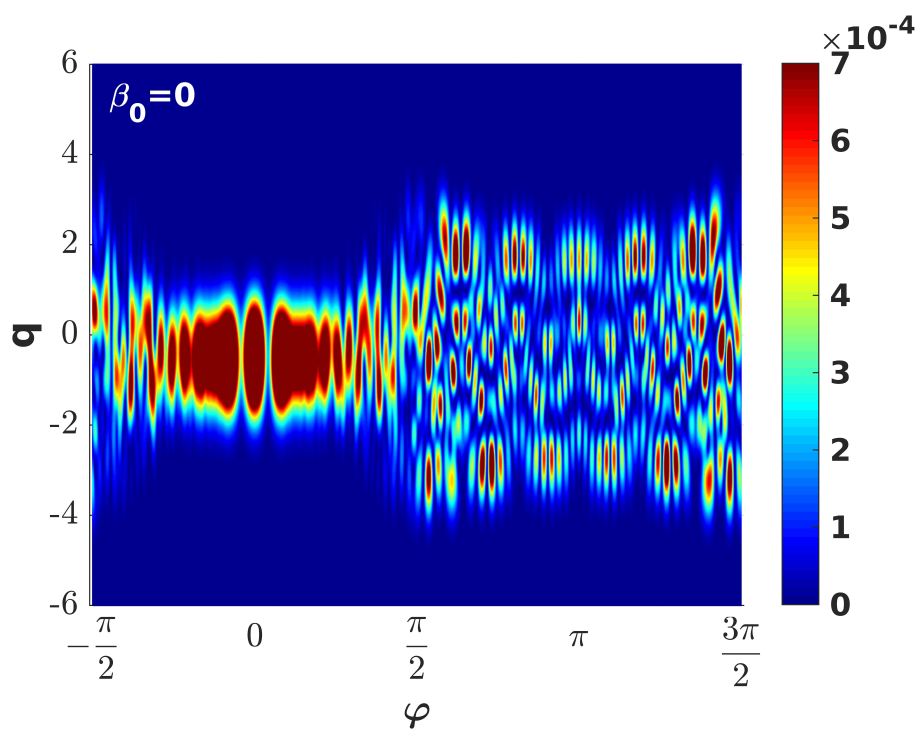

Figure 3: The probability density for the wave packet excited by the transform-limited pulse $\left(\beta_{0}=0\right)$ at $t=250$ fs. For $\varphi<\pi / 2$ we have the excited-state cis configuration whereas the ground-state trans configuration corresponds to $\varphi>\pi / 2$.

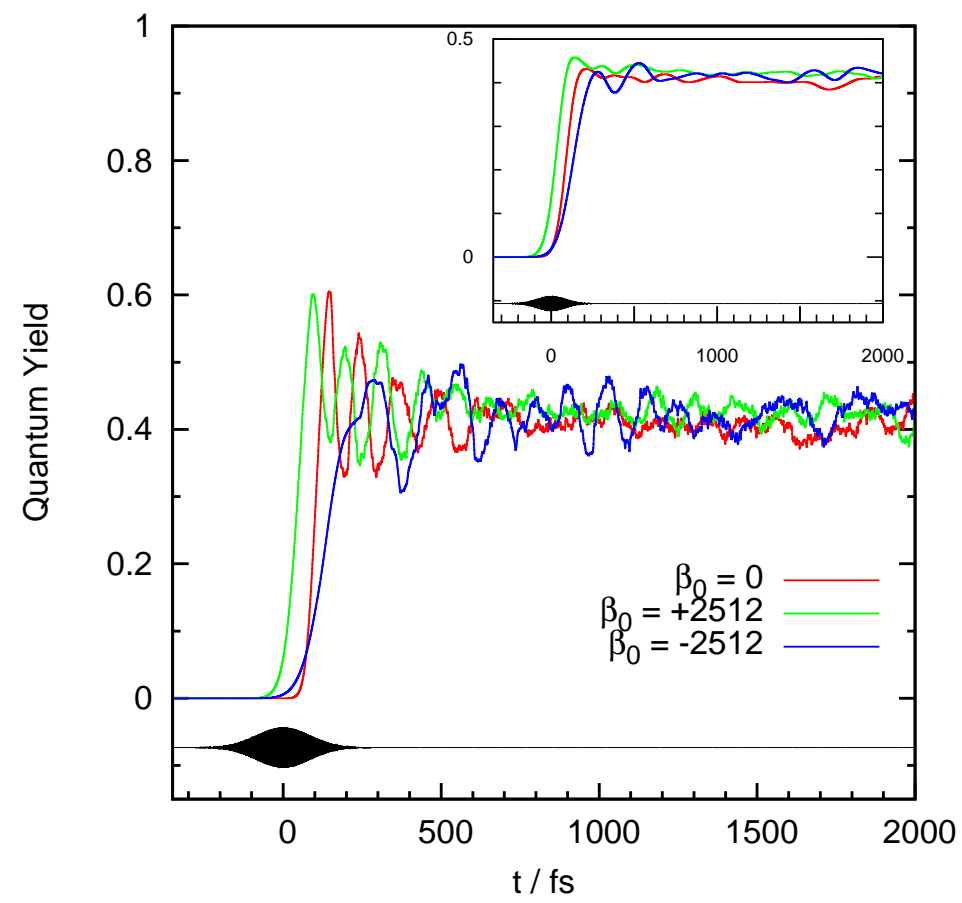

Figure 4: The quantum yield of the trans isomer calculated with the two-dimensional Hamiltonian including dissipation $\lambda_{d}=0.05$. The yield is shown for the transform-limited pulse $\left(\beta_{0}=0\right)$ as well as for two chirped pulses with the electric field peaked at $t=0$. The inset shows the yields convoluted with a 100 fs (FWHM) Gaussian probe pulse. 
on the chirp cannot be identified.

Figure 5 shows the quantum yield for the stronger dampening $\lambda_{d}=0.5$, again convoluted with a 100 fs (FWHM) Gaussian probe pulse. We observe that the larger dampening leads

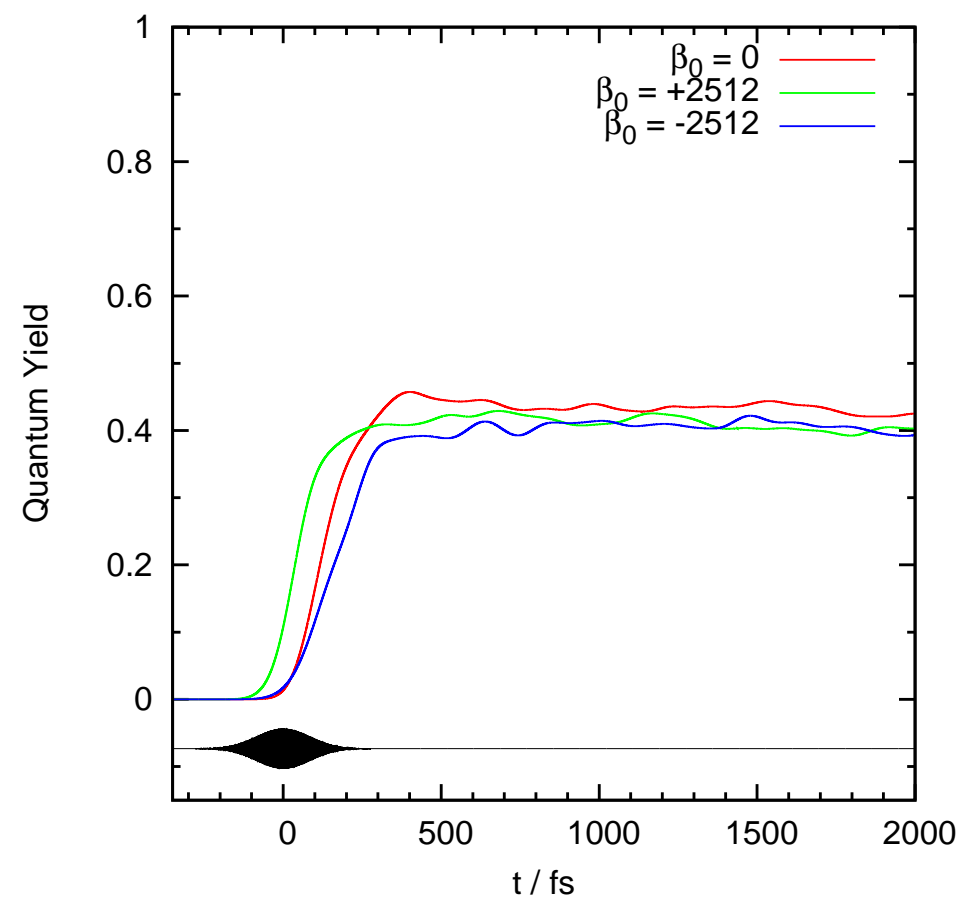

Figure 5: The quantum yield of the trans isomer calculated with the two-dimensional Hamiltonian including dissipation $\lambda_{d}=0.5$. The yields are convoluted with a 100 fs (FWHM) Gaussian probe pulse and shown for the transform-limited pulse $\left(\beta_{0}=0\right)$ as well as for two chirped pulses with the electric field peaked at $t=0$.

to a faster stabilization of the branching ratio but it hardly affects the final quantum yield. This is in agreement with previous work in the Redfield description for the system-bath coupling. ${ }^{24}$ After $300 \mathrm{fs}$, the quantum yield for the unchirped pulse is larger than the yields for the chirped pulses and when the yields have stabilized for times longer than about $1.5 \mathrm{ps,}$ it is observed that the quantum yield for the unchirped pulse is about $5 \%$ higher compared to the chirped pulses. This demonstrates coherent control in the weak-field limit. We could not identify larger effects in the present model. A similar small dependence on laser phase was suggested by a computational study of one-photon phase control of the cis-trans isomerization in retinal. ${ }^{30}$ It is interesting to note that the time dependence in Figure 5 is very similar to 
that obtained from a description of the reaction kinetics of cis $\rightleftarrows$ trans isomerization.

Concerning the mechanism leading to a dependence on the laser chirp, we note that from the topology of the potential energy surface (see Figures 1 and 2) it is clear that a prompt stabilization of the (ground-state) trans configuration is possible, i.e., to trap this part of the wave packet below the barrier for the trans-cis isomerization. On the other hand, the part of the wave packet corresponding to the excited-state cis configuration cannot be directly trapped below the barrier for trans-cis isomerization but, at best, at the conical intersection or indirectly to the ground-state cis configuration via transfer through the conical intersection. Thus, the cis/trans populations of the adiabatic ground state cannot settle before the excited-state $\left(S_{1}\right)$ cis population has fully decayed. This explains why the effect of chirp is small in the present case.

Finally, we note that a recent experimental study on retinal photoisomerization used transform-limited pulses with durations $\tau_{0} \sim 20$ fs leading to chirped pulses with durations of the order of 2 ps. ${ }^{18}$ We found that for the mechanism discussed above, such pulses are too long compared to the timescale of dissipation.

Summary. Substantial weak-field coherent control has been predicted for a simple onedimensional model of isomerization where the two isomers are represented by two nonadiabatically coupled Morse potentials. ${ }^{29}$ The opportunity for control emerges when environmentally assisted energy relaxation is sufficiently fast, such that, the phase-shaped wave packet can be trapped below the barrier for isomerization (i.e., the crossing of the Morse potentials). In that model, both isomers are described only by bound states. This facilitates the prompt trapping of the isomers.

We have investigated a similar mechanism for weak-field (one-photon) coherent control of cis-trans photoisomerization using a well-established model Hamiltonian. Thus, we have studied whether different phase-shaped wave packets, corresponding to different instantaneous yields, can be quickly stabilized ("dumped") - with some selectivity - in the trans configuration due to prompt energy dissipation. We have presented a detailed investigation 
of this mechanism and did find evidence for phase control in the model given sufficiently strong dampening. The addition of quantum entanglement between the system and the bath can further influence the outcome. We note, however that the magnitude of our predicted phase effect is similar to what has been suggested by a computational study using the MCTDH approach for a similar model Hamiltonian including explicit "bath modes". ${ }^{30}$

The smallness of the phase effect for the photoisomerization described in the present work will obviously make experimental verifications challenging, the identification of a suitable molecule and solvent allowing for prompt energy dissipation seems to be crucial.

\section{Acknowledgement}

The research leading to these results has received funding from the European Union's Horizon 2020 research and innovation programme under the Marie Sklodowska-Curie Grant agreement No. 713683 (COFUNDfellowsDTU).

\section{References}

(1) Rice, S.A.; Zhao, M. Optical Control of Molecular Dynamics; Wiley, New York, 2000.

(2) Henriksen, N.E. Laser Control of Chemical Reactions. Chem. Soc. Rev. 2002, 31, 37-42.

(3) Brif, C.; Chakrabarti, R.; Rabitz, H. Control of Quantum Phenomena: Past, Present and Future. New J. Phys. 2010, 12, 075008.

(4) Shapiro, M.; Brumer, P. Quantum Control of Molecular Processes; Wiley, New York, 2012.

(5) Worth, G.A.; Sanz, C.S. Guiding the Time-Evolution of a Molecule: Optical Control by Computer. Phys. Chem. Chem. Phys. 2010, 48, 15570-15579. 
(6) Engel, V.; Meier, C.; Tannor, D.J. Local Control Theory: Recent Applications to Energy and Particle Transfer Processes in Molecules. Adv. Chem. Phys. 2009, 141, $29-101$.

(7) Saab, M.; Doriol, L.J.; Lasorne, B.; Guérin, S.; Gatti, F. A Quantum Dynamics Study of the Benzopyran Ring Opening Guided by Laser Pulses. Chem. Phys. 2014, 442, 93-102.

(8) Liu, C.; Manz J.; Ohmori, K.; Sommer, C.; Nobuyuki, T.; Tremblay, J.C.; Zhang, Y. Attosecond Control of Restoration of Electronic Structure Symmetry. Phys. Rev. Lett. 2018, 121, 173201.

(9) Brumer, P.; Shapiro, M. One Photon Mode Selective Control of Reactions by Rapid or Shaped Laser Pulses: An Emperor Without Clothes? Chem. Phys. 1989, 139, 221-228.

(10) Shapiro, M.; Brumer, P. On the Origin of Pulse Shaping Control of Molecular Dynamics. J. Phys. Chem. A 2001, 105, 2897-2902.

(11) Spanner, M.; Arango, C.A.; Brumer, P. Communication: Conditions for One-Photon Coherent Phase Control in Isolated and Open Quantum Systems. J. Chem. Phys. 2010, $133,151101$.

(12) García-Vela, A.; Henriksen, N.E. Coherent Control of Photofragment Distributions Using Laser Phase Modulation in the Weak-Field Limit. J. Phys. Chem. Lett. 2015, 6, 824-829.

(13) Shu, C.C.; Henriksen, N.E. Coherent Control of Indirect Photofragmentation in the Weak-Field Limit: Control of Transient Fragment Distributions. J. Chem. Phys. 2011, 134, 164308.

(14) Tiwari, A.K.; Dey, D.; Henriksen, N.E. Laser-Pulse-Shape Control of Photofragmentation in the Weak-Field Limit. Phys. Rev. A 2014, 89, 023417. 
(15) Tiwari, A.K.; Henriksen, N.E. Phase-Only Laser Control in the Weak-Field Limit: Two-Pulse Control of IBr Photofragmentation Revisited. J. Chem. Phys. 2016, 144, 014306 .

(16) Dey, D.; Tiwari, A.K.; Henriksen, N.E. Coherent Control of Selective Bond Breaking: HOD in the $\tilde{A}$-state Revisited. Chem. Phys. Lett. 2019, 716, 131-133.

(17) Prokhorenko, V.I.; Nagy, A.M.; Waschuk, S.A.; Brown, L.S.; Birge, R.R.; Dwayne Miller, R.J. Coherent Control of Retinal Isomerization in Bacteriorhodopsin. Science 2006, 313, 1257-1261.

(18) Liebel, M.; Kukura, P. Lack of Evidence for Phase-Only Control of Retinal Photoisomerization in the Strict One-Photon Limit. Nat. Chem. 2017, 9, 45-49.

(19) Wang, Q.; Schoenlein, R.W.; Peteanu, L.A.; Mathies, R.A., Shank, C.V. Vibrationally Coherent Photochemistry in the Femtosecond Primary Event of Vision. Science 1994, 266, $422-424$.

(20) Seidner, L.; Domcke, W. Microscopic Modelling of Photoisomerization and InternalConversion Dynamics. Chem. Phys. 1994, 186, 27-40.

(21) Seidner, L.; Stock, G.; Domcke, W. Nonperturbative Approach to Femtosecond Spectroscopy: General Theory and Application to Multidimensional Nonadiabatic Photoisomerization Processes. J. Chem. Phys. 1995, 103, 3998-4011.

(22) Hahn, S.; Stock, G. Quantum-Mechanical Modeling of the Femtosecond Isomerization in Rhodopsin. J. Phys. Chem. B 2000, 104, 1146-1149.

(23) Hahn, S.; Stock, G. Femtosecond Secondary Emission Arising from the Non-adiabatic Photoisomerization in Rhodopsin. Chem. Phys. 2000, 259, 297-312.

(24) Hahn, S.; Stock, G. Ultrafast Cis-Trans Photoswitching: A Model Study. J. Chem. Phys. 2002, 116, 1085-1091. 
(25) Balzer, B.; Stock, G. Transient Spectral Features of a Cis-Trans Photoreaction in the Condensed Phase: A Model Study. J. Phys. Chem. A 2004, 108, 6464-6473.

(26) Flores, S.-C.; Batista, V.S. Model Study of Coherent-Control of the Femtosecond Primary Event of Vision. J. Phys. Chem. B 2004, 108, 6745-6749.

(27) Abe, M.; Ohtsuki, Y.; Fujimura, Y.; Domcke, W. Optimal Control of Ultrafast CisTrans Photoisomerization of Retinal in Rhodopsin via a Conical Intersection. J. Chem. Phys. 2005, 123, 144508.

(28) Hoki, K.; Brumer, P. Dissipation Effects on Laser Control of Cis/Trans Isomerization. Chem. Phys. Lett. 2009, 468, 23-27.

(29) Katz, G.; Ratner, M.A.; Kosloff, R. Control by Decoherence: Weak Field Control of an Excited State Objective. New J. Phys. 2010, 12, 015003.

(30) Arango, C.A.; Brumer, P. Communication: One-Photon Phase Control of Cis-Trans Isomerization in Retinal. J. Chem. Phys. 2013, 138, 071104.

(31) Henriksen, N.E.; Møller, K.B. Instantaneous Nonvertical Electronic Transitions with Shaped Femtosecond Laser Pulses: Is it Possible? J. Chem. Phys. 2003, 119, 25692576 .

(32) Nest, M.; Meyer, H.-D. Dissipative Quantum Dynamics of Anharmonic Oscillators with the Multiconfiguration Time-Dependent Hartree Method. J. Chem. Phys. 2003, 119, 24-33.

(33) Kohen, D.; Tannor, D.J. Classical-Quantum Correspondence in the Redfield Equation and its Solutions. J. Chem. Phys. 1997, 107, 5141-5153.

(34) Am-Shallem, M.; Kosloff, R. The Scaling of Weak Field Phase-Only Control in Markovian Dynamics. J. Chem. Phys. 2014, 141, 044121. 
(35) Gerber, R.B.; Buch, V.; Ratner, M.A. Time-Dependent Self-Consistent Field Approximation for Intramolecular Energy Transfer. I. Formulation and Application to Dissociation of van der Waals Molecules. J. Chem. Phys. 1982, 77, 3022-3030.

(36) Kotler, Z.; Neria, E.; Nitzan, A. Multiconfiguration Time-Dependent Self-Consistent Field Approximations in the Numerical Solution of Quantum Dynamical Problems. Comp. Phys. Comm. 1991, 63, 243-258.

(37) Kosloff, R.; Tal-Ezer, H. A Direct Relaxation Method for Calculating Eigenfunctions and Eigenvalues of the Schrödinger Equation on a Grid. Chem. Phys. Lett. 1986, 127, 223-230.

(38) Manescu, C.; Krause, J.L.; Møller, K.B.; Henriksen, N.E. Suppressing the Spreading of Continuum Wave Packets via Chirped Laser Pulses. J. Phys. Chem. A 2004, 108, $8840-8847$.

(39) Feit, M.D.; Fleck Jr., J.A.; Steiger, A. Solution of the Schrödinger Equation by a Spectral Method. J. Comput. Phys. 1982, 47, 412-433.

(40) Kosloff, D.; Kosloff, R. A Fourier Method Solution for the Time Dependent Schrödinger Equation as a Tool in Molecular Dynamics. J. Comput. Phys. 1983, 52, 35-53.

(41) Corey, G.C.; Lemoine, D. Pseudospectral Method for Solving the Time-Dependent Schrödinger Equation in Spherical Coordinates. J. Chem. Phys. 1992, 97, 4115-4126.

(42) Tannor, D.J. Introduction to Quantum Mechanics: A Time-Dependent Perspective; University Science Books, Sausalito, CA, 2007. 